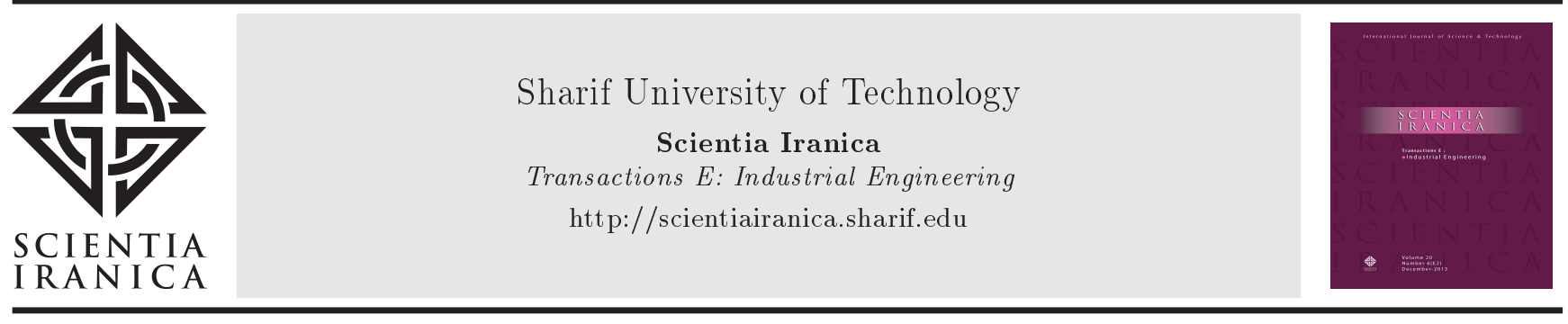

\title{
Finding an improved region of efficiency via DEA-efficient hyperplanes
}

\author{
N. Ebrahimkhani Ghazi ${ }^{a}$, F. Hosseinzadeh Lotfi ${ }^{a}{ }^{*}$, M. Rostamy-Malkhalifeh ${ }^{a}$, \\ G.R. Jahanshahloo ${ }^{\mathrm{b}}$, and M. Ahadzadeh Namin ${ }^{\mathrm{c}}$ \\ a. Department of Mathematics, Science and Research Branch, Islamic Azad University, Tehran, Iran. \\ b. Department of Mathematics, Teacher Training University, Tehran, Iran. \\ c. Department of Mathematics, Shahr-e-Qods Branch, Islamic Azad University, Tehran, Iran.
}

Received 13 December 2016; received in revised form 2 June 2017; accepted 18 September 2017

\section{KEYWORDS}

Data Envelopment Analysis (DEA);

Efficiency;

Value judgment;

Linear programming;

Production Possibility

Set (PPS)

\begin{abstract}
The analysis of efficiency is conducted for two vital purposes: firstly, in order to evaluate the current level of efficiency; secondly, to provide information on how to improve the level of efficiency, which is to provide benchmarking information. The inefficient Decision Making Units (DMUs) are usually able to improve their performance, and Data Envelopment Analysis (DEA) projections provide a prescription for improvement. However, sometimes, an inefficient DMU cannot move its performance toward the best practice by either decreasing its inputs or increasing its outputs. On the other hand, it can scarcely reach its efficient benchmark. This research suggests a method to find an improved region of efficiency through DEA-efficient hyperplanes by providing an algorithm for detecting an improved efficiency path. In addition to the production of reasonable benchmarking information, the proposed algorithm provides the general requirements that satisfy the demands which every professional decision-maker should meet. Finally, we provide a more detailed description of some new issues, extending the insights from this analysis of the benchmark region from the under-evaluated inefficient DMU. Finally, numerical examples are provided to demonstrate the results of the analysis.
\end{abstract}

(C) 2018 Sharif University of Technology. All rights reserved.

\section{Introduction}

The traditional Data Envelopment Analysis, as initially proposed by Charnes et al. [1], is a relatively new "data-oriented" approach to assessing the performance of a set of DMUs that use multiple inputs to produce multiple outputs. Most DEA approaches, such as the approach introduced by [2], focus on relative efficiencies.

*. Corresponding author.

E-mail addresses: nasrinebrahimkhanighazi2012@gmail.com (N. Ebrahimkhani Ghazi); farhad@hosseinzadeh.ir (F. Hosseinzadeh)

doi: $10.24200 /$ sci. 2017.20004
Recently, some researchers have studied the performance improvement in DEA to meet the decisionmaker's goals [3-6]. So many DEA-efficiency improvement papers have certain points of view such as the modified formulation of the DEA model where bounds are imposed on each factor; this model was proposed by Kao [7]. Another model was introduced by Chen et al. [8] that modified the original DEA model by decomposing the normalizing equation for being associated with the different dual variables. In addition, by means of the equation of defining hyperplanes of PPS, one can find the region of efficiency improvement. In 2011, Khoshfetrat and Daneshvar [9], in a paper, proposed improving the weak efficiency frontiers in fuzzy DEA models, where they implicitly dealt with the knowledge of the sensitivity analysis in depth. 
This may indeed show the importance of obtaining the defining hyperplanes of the PPS.

The studies of efficient frontiers and characterization of different types of hyperplanes have been less intensively investigated $[10,11]$. The connotation of the efficient frontier DMUs in the DEA was studied by Jahanshahloo et al. [12]; this study found the piecewise linear frontier of the production function. In the aforementioned study, the researchers developed a way to obtain efficient frontier using 0-1 integer programming. Despite the greater computational efforts required to solve the $0-1$ integer programming for small $n$ (number of DMUs), solving the 0-1 model for large $n$ is preferable to solving $n$ linear programs; these preference relationships can be derived as strengths. In addition, searching for the efficient frontier in the DEA was considered by Korhonen [13]. Their approach was developed to create a free search for the efficient frontier in Multiple-Objective Linear Programming (MOLP) MOLP-this can also be used in DEA. Furthermore, Jahanshahloo et al. [14] suggested an approach to finding strong defining hyperplanes of the PPS in DEA, in which the observed deficiency of an alternative optimal solution of a multiplier form has been removed. In addition, the construction of all DEA-efficient frontiers in Generalized Data Envelopment Analysis (GDEA) was discussed by $\mathrm{Yu}$ and Zeleny [15]. In their work, they derived a necessary and sufficient condition for a point to be nondominated. It provided a simple proof confirming that the set of all nondominated extreme points is indeed connected. They then obtained a decomposition theorem and some necessary and sufficient conditions for a face to be nondominated. Lotfi et al. [16] provided an algorithm in order to find efficient hyperplanes with the Variable Returns to Scale (VRS) assumption of technology by using the MOLP structure. They characterized the efficient hyperplanes that make up the DEA efficient frontier only by finding the efficient faces of the MOLP problem without exploring its extreme efficient points. Sensitivity and stability analysis of the specific underevaluated DMU is one of the areas of interest in DEA. In 1985, Charnes et al. [17] initiated the sensitivity analysis of a CCR model for a specific efficient DMU with a single output. They constructed variations in the data for the DMU under consideration and initiated the change in the inverse matrix used to produce solutions in typical simplex algorithm computer codes. Then, in 2015, Forghani et al. considered sensitivity analysis in the two-stage DEA, and found that the necessary and sufficient conditions for preserving a DMU's efficiency classification were developed when various data changes were applied to all the DMUs [18]. Many more papers have studied sensitivity analysis with various data types such as integer data type [3], fuzzy data [19], etc. Another model proposed by
Huang et al. [20], later generalized by Jahanshahloo et al. [21], showed that all the efficient surfaces of the $\mathrm{BCC}$ model passing through $\mathrm{DMU}_{P}$ were generated for the case; in this model, the largest stability region of the under-evaluated extreme efficient DMU was constructed by the omission of $\mathrm{DMU}_{P}$ from the observations set. Later, the largest stability region proposed in their paper was improved by [22]. Today, many algorithms have been proposed to find the efficient region of a specified DMU simply specified by the concept of all the possible values that remain or become efficient $[23,24]$.

DEA also provides benchmarking information in order to improve the efficiency of the DMU. This information distinguishes DEA from other efficiency methodologies. The reference points that are used for obtaining the benchmarking information are vital not only for evaluating the potential performance of the DMU, but also for providing information on how to improve its performance, as observed by Bogetoft and Hougaard [5]. Inappropriate selection of reference points might lead to misleading guidance on how to improve efficiency. The DEA model was introduced by Farrell [25], and later developed by Charnes et al. [1]; it uses an oriented radial measure of efficiency for the identification of reference points on the boundary of the PPS with the input orientation or the output orientation of the observed unit. The main weakness of radial efficiency measures is that they disregard the possible existence of slacks associated with the projected points on the production frontier (see the following examples: $[9,19,23,26])$. Many practical studies have used non-oriented measures; for example, Colwell and Davis [27] used an intermediation approach that specified inputs in the form of costs and outputs in the form of revenue. Finding the closest target has been considered for both oriented models (see the following examples: [28-30]) and non-oriented models (see the following examples: [31-35]). The only question that most DEA studies focus on is "how is a company inefficient?"; the question of "how can a company become efficient?" is the second case for review. Interactive DEA (IDEA) usage was suggested by Post and Spronk [32] in order to make the benchmarking information sensible from the inefficient DMU's viewpoint. In addition, for obtaining the most relevant and easily attainable benchmarking information, the leastdistance measure was proposed by Baek and Lee [36], which provides a well-defined efficiency measure, too. To answer the second question, this study focuses on the best rational way for an inefficient DMU to meet the DM's needs, whether it is still inefficient or not. On the flip side, the simplistic claim that the region of efficiency improvement is more relevant than finding their efficiency is really misleading. Applying the results of $[16,20-22,27,38]$, the current paper aims 
to obtain the improved region of efficiency by using all the defining hyperplanes of PPS. Now, there is a need to evaluate the use of DEA as a decision aid for considering the improved region of efficiency based on the overall value judgment; this is clarified in [39]. The value judgment here can, therefore, be expressed in our new proposed algorithm to describe the benchmarking region improvement concept. By means of the new algorithm, the improvement path for an inefficient DMU based on value judgment is thereafter identified. In measuring the improvement region, the incorporation of value judgment is, therefore, also very important. The remaining parts of this paper will be unfolded as follows. To explain the new benchmarking region, we start with a description of obtaining the defining hyperplanes of PPS in Section 2, which briefly reviews a mathematical basis used for this study. In Section 3, we illustrate our proposed algorithm to obtain the improvement of the benchmarking region. In Section 4, three numerical examples are documented for intuitively describing the new algorithm in order to find an improved region of efficiency. Section 5 provides our concluding remarks and future directions for research.

\section{Preliminaries}

In this section, a brief description of the BCC inputoriented model and the approaches to finding efficient hyperplanes are included.

\subsection{The BCC input-oriented model}

The DEA-BCC model was proposed for dealing with situations whose proportionality between the inputs and outputs is not constant along the efficient frontier. The BCC model [4] was initially developed by adding a convexity restriction to the CCR [1] model envelope formulation. This generated a VRS frontier. Suppose that we have $n$ DMUs, where each $\operatorname{DMU}_{j}(j=1,2, \cdots, n)$ produces the same $s$ outputs in (possibly) different amounts, $y_{r j}(r=1,2, \cdots, s)$, using the same $m$ inputs, and $x_{i j}(i=1,2, \cdots, m)$ in (possibly) different amounts. The BCC input-oriented model evaluates the efficiency of $\mathrm{DMU}_{o}$, the DMU under consideration, by solving the following "multiplier linear form" program:

$$
\begin{array}{ll}
\max & \sum_{r=1}^{s} u_{r} y_{r o}-u_{o}, \\
\text { s.t. } & \sum_{i=1}^{m} v_{i} x_{i o}=1, \\
& \sum_{r=1}^{s} u_{r} y_{r j}-\sum_{i=1}^{m} v_{i} x_{i o}-u_{o} \leq 0,
\end{array}
$$

$$
\begin{aligned}
& j=1, \cdots, n, \quad u_{o}, \text { free, } \\
& v_{i}, u_{r} \geq \varepsilon>0, \\
& i=1,2, \cdots, m, \quad r=1,2, \cdots, s,
\end{aligned}
$$

where $u_{i}$ and $v_{i}$ are the decision variables, respectively, that are associated with output $r$ and input $i$, and $\varepsilon$ is a positive non-Archimedean infinitesimal.

In addition to the efficiency score, the most practical information given by the DEA study is the set of Pareto-efficient projection points for the underevaluated DMU. The coordination of a projection point can be interpreted as the "target" levels of performance of the inputs and outputs. The obtained targets provide an indication of how an assessed DMU improves its performance efficiently. Therefore, the closer the assessed DMU is to the targets, the lesser practical effort it needs to be efficient. The basic DEA model in [1], which is called the CCR model, has led to several extensions, most notably the BCC model of [4]. Assume that there are $n \mathrm{DMU}_{s}\left(\mathrm{DMU}_{j}\right.$ : $j=1,2, \cdots, n)$ that consume $X_{j}=\left(x_{1 j}, \cdots, x_{m j}\right)$ to produce $Y_{j}=\left(y_{1 j}, \cdots, y_{s j}\right)$. The BCC input-oriented (BCC-I) model evaluates the efficiency of $\mathrm{DMU}_{o}$ and the considered DMU by solving the following linear program:

$$
\begin{aligned}
& \min \theta \text {, } \\
& \text { s.t. } \quad \sum_{j} \lambda_{j} x_{i j} \leq \theta x_{i o} ; \quad \forall i, \\
& \sum_{j} \lambda_{j} y_{r j} \geq y_{r o} ; \quad \forall r \\
& \sum_{j} \lambda_{j}=1 ; \quad \forall j, \\
& \lambda_{j} \geq 0 ; \quad \forall j,
\end{aligned}
$$

where $\theta^{*}$ is the optimal solution obtained from Eq. (2).

Definition 1: $\left(\sum_{j=1}^{n} \lambda_{j}^{*} x_{j}, \sum_{j=1}^{n} \lambda_{j}^{*} y_{j}\right)=\left(\theta^{*} x_{o}, y_{o}\right)$ is the input-oriented projection point of $\mathrm{DMU}_{o}$ on the efficient frontier.

\subsection{Finding efficient hyperplanes}

A method for generating all the efficient surfaces of the $\mathrm{BCC}$ model passing through $\mathrm{DMU}_{p}$ was suggested by $[20,38]$ and improved by [22], which are as follows:

$$
\begin{array}{ll}
\min & u_{o}, \\
\text { s.t. } & \sum_{r=1}^{s} u_{r} y_{r p}-\sum_{i=1}^{m} v_{i} x_{i p}=u_{o},
\end{array}
$$




$$
\begin{aligned}
& \sum_{r=1}^{s} u_{r} y_{r j}-\sum_{i=1}^{m} v_{i} x_{i j} \leq u_{o}, \\
& \quad j=1, \cdots, n \\
& \sum_{i=1}^{m} v_{i}+\sum_{r=1}^{s} u_{r}=1, \\
& v_{i} \geq \varepsilon, \quad u_{r} \geq \varepsilon, \\
& \quad i=1,2, \cdots, m, \quad r=1,2, \cdots, s,
\end{aligned}
$$

$\max u_{o}$

$$
\begin{array}{ll}
\text { s.t. } \quad & \sum_{r=1}^{s} u_{r} y_{r p}-\sum_{i=1}^{m} v_{i} x_{i p}=u_{o}, \\
& \sum_{r=1}^{s} u_{r} y_{r j}-\sum_{i=1}^{m} v_{i} x_{i j} \leq u_{o}, \\
j=1,2, \cdots, n, \\
\sum_{i=1}^{m} v_{i}+\sum_{r=1}^{s} u_{r}=1, \\
v_{i} \geq \varepsilon, \quad u_{r} \geq \varepsilon, \quad r=1,2, \cdots, s .
\end{array}
$$

Note that in an optimal solution to Relations (3) and (4), all of those observed efficient points that satisfy their respective constraints as equalities also lie on the efficient facet contained in the hyperplane passing through $\left(x_{p}, y_{p}\right)$. To conduct sensitivity analysis of each efficient unit called $\mathrm{DMU}_{p}$, Jahanshahloo et al. [21] used defining hyperplanes that are binding at $\mathrm{DMU}_{p}$ and the new frontier, which is formed by the omission $\mathrm{DMU}_{p}$ from the observations set.

Moreover, the MOLP problem suggested by [16] for finding efficient hyperplanes with the VRS assumption of technology in DEA is as follows:

$$
\begin{array}{ll}
\max \quad\left\{U Y_{1}-V X_{1}-u_{o}, U Y_{2}-V X_{2},\right. & \\
& \left.-u_{o}, \cdots, U Y_{n}-V X_{n}-u_{o}\right\}, \\
\text { s.t. } \quad & U Y_{j}-V X_{j}-u_{o} \leq 0, \quad j=1, \cdots, n, \\
& 1 U+1 V=1, \\
& V \geq 1 \varepsilon, \quad U \geq 1 \varepsilon .
\end{array}
$$

Changing the inequality constraints of Model (5) to equalities and minimizing the objective function of the converted model are as follows:

$$
\begin{array}{ll}
\min & \left\{S_{1}, S_{2}, \cdots, S_{n}\right\}, \\
\text { s.t. } & U Y_{j}-V X_{j}-u_{o}+S_{j}=0, \quad j=1, \cdots, n, \\
& 1 U+1 V=1, \\
& S_{j} \geq 0, \quad j=1, \cdots, n, \\
& V \geq 1 \varepsilon, \quad U \geq 1 \varepsilon .
\end{array}
$$

Assume that $J=\{1, \cdots, n\}$ and $M=\{I \mid I \subseteq J\}$. The feasible region of Model (6) is denoted by $W$ For $I \in M ; F(I)$ represents a face of $W[15]$. Note that $F(\varnothing)=W$; for $I \in M, F(I)=\varnothing$ is possible. Model $\left(S P_{I}\right)$, which is similar to the model proposed by [37], is as follows:

$$
\begin{aligned}
& Z_{I}=\max \sum_{j=1}^{n} T_{j}-\sum_{j=1}^{n} S_{j}, \\
& \text { s.t. } U Y_{j}-V X_{j}-u_{o}+S_{j}=0 \text {, } \\
& j=1, \cdots, n, \\
& U^{\prime} Y_{j}-V^{\prime} X_{j}-u_{o}^{\prime}+T_{j}=0 \\
& j=1, \cdots, n, \\
& S_{j}-T_{j} \leq 0, \quad j=1, \cdots, n, \\
& T_{j}=0, \quad j \in I, \\
& 1 U+1 V=1 \\
& 1 U^{\prime}+1 V^{\prime}=1 \\
& S_{j} \geq 0, \quad T_{j} \geq 0, \quad j=1, \cdots, n, \\
& U \geq 1 \varepsilon, \quad U^{\prime} \geq 1 \varepsilon \\
& V \geq 1 \varepsilon, \quad V^{\prime} \geq 1 \varepsilon .
\end{aligned}
$$

The algorithm for finding efficient hyperplanes with the VRS technology in DEA, which was proposed by Hosseinzadeh et al. [16], is as follows:

Step 1. Let $k=1, F^{k}=\varnothing, E F^{k}=\varnothing$, and $J=\{1,2, \cdots, n\}$. Subscripted sets $F^{k}$ and $E F^{k}$ are the set of DMUs on the faces of the PPS and the set of subscripts of the DMUs on efficient faces of the PPS, respectively. With the VRS assumption of technology, dictating that both sets of DMUs contain $k$-element sets, proceed to Step 2;

Step 2. Evaluate $M^{k}=\{I \subseteq J|| I \mid=k\}$. If $|J|<k$ and $k=n+1$, then stop. Otherwise, go to Step 3; 
Step 3. If $M^{k} \neq \varnothing$, select $I \in M^{k}$; then, proceed to Step 4. Otherwise, go to Step 5;

Step 4. Solve $S P_{I}$ and set $M^{k}=M^{k} \backslash I$. If $Z_{I}>0$, then $F^{k}=F^{k} \cup I$ and proceed to Step 3. If $Z_{I}=0$, then $F^{k}=F^{k} \cup I$ and $E F^{k}=E F^{k} \cup I$. Suppose that $\left(U^{*}, V^{*}, u_{o}^{*}\right)^{k}$ is the optimal solution of $S P_{I}$ as the gradients of the efficient hyperplanes. If $S P_{I}$ is infeasible, proceed to Step 3;

Step 5. Let $k=k+1, F^{k}=\varnothing, E F^{k}=\varnothing$, and $\cup_{I \in F^{k}} I=J$. Proceed to Step 2 .

Owing to the finite number of DMUs and the feasibility of linear programming problem $S P_{I}$, the iterative proposed algorithm is guaranteed to converge.

Another approach to identifying the equations of the weak defining hyperplanes of the PPS of the BCC model was proposed by Jahanshahloo et al. [38]. The summary of this identification is as follows:

Step 1. Considering each $\mathrm{DMU}_{k}(k \in J)$ evaluate the following models:

$$
\begin{array}{ll}
\min & \theta_{l}^{k}-\varepsilon\left(\sum_{i=1}^{m} S_{i}^{-}+\sum_{r=1}^{s} S_{r}^{+}\right), \\
\text {s.t. } \quad & \sum_{j \in J-\{k\}} \lambda_{j}^{k} x_{l j}+S_{l}^{-}=\theta_{l}^{k} x_{l k}, \\
& \sum_{j \in J-\{k\}} \lambda_{j}^{k} x_{i j}+S_{i}^{-}=x_{i k}, \\
& i=1, \cdots, m, \quad i \neq l, \\
& \sum_{j \in J-\{k\}} \lambda_{j}^{k} y_{r j}-S_{r}^{+}=y_{r k}, \\
& r=1, \cdots, s, \\
& \sum_{j \in J-\{k\}} \lambda_{j}^{k}=1, \quad \\
& \lambda_{j}^{k} \geq 0 \quad(j \in J=\{1, \cdots, n\})-\{k\}, \\
& S_{i}^{-} \geq 0, \quad S_{l}^{-} \geq 0, \quad r=1, \cdots, s, \\
& l=1, \cdots, m, \\
& S_{r}^{+} \geq 0 \quad i=1, \cdots, m, \quad \theta_{l}^{k} \text { free. }
\end{array}
$$

$\max \varphi_{q}^{k}+\varepsilon\left(\sum_{i=1}^{m} t_{i}^{-}+\sum_{r=1}^{s} t_{r}^{+}\right)$ s.t. $\quad \sum_{j \in J-\{k\}} \mu_{j}^{k} x_{i j}+t_{i}^{-}=x_{i k}, \quad i=1, \cdots, m$,

$$
\begin{aligned}
& \sum_{j \in J-\{k\}} \mu_{j}^{k} y_{q j}-t_{q}^{-}=\varphi_{q}^{k} y_{q k}, \\
& \sum_{j \in J-\{k\}} \mu_{j}^{k} y_{r j}-t_{r}^{+}=y_{r k}, \\
& \quad r=1, \cdots, s, \quad r \neq q, \\
& \sum_{j \in J-\{k\}} \mu_{j}^{k}=1, \\
& \mu_{j}^{k} \geq 0, \quad j \in J-\{k\}, \\
& t_{i}^{-} \geq 0, \quad i=1, \cdots, m, \\
& t_{r}^{+} \geq 0, \quad r=1, \cdots, s, \quad q=1, \cdots, s, \\
& \varphi_{q}^{k} \text { free. }
\end{aligned}
$$

in which $\varepsilon$ is a non-Archimedean number;

Step 2. Form two sets $I_{l}$ and $O_{q}$. Corresponding with any $\mathrm{DMU}_{j} \in I_{l}$ (or $O_{q}$ ), form the weak efficient virtual, $\mathrm{DMU}_{j}^{l}\left(\mathrm{DMU}_{j}^{m+q}\right)$, as mentioned previously. Put the indices of the extreme efficient and weak efficient virtual DMUs in $F$. Let $|F|=L$;

Step 3. For each $p, q \in F$ in which $p \neq q$, evaluate $\mathrm{DMU}_{k}=\frac{1}{2} \mathrm{DMU}_{p}+\frac{1}{2} \mathrm{DMU}_{q}$; if it is efficient, then $p \in F_{q}$ and $q \in F_{p}$;

Step 4. For each $(j=1, \cdots, L), \bar{F}_{j}=F-F_{j}$;

Step 5. Choose the arbitrary $m+s$ members of $F$ so that none of them belongs to another $\bar{F}$. Call this set $D=\left\{j_{1} \cdots \cdot j_{m+s}\right\}$. Using $D$, a hyperplane can be constructed as follows:

$$
\left|\begin{array}{ccc}
x_{1}-x_{1 j_{1}} & \cdots & x_{m}-x_{m j_{1}} \\
x_{1 j_{2}}-x_{1 j_{1}} & \cdots & x_{m j_{2}}-x_{m j_{1}} \\
\cdot & \cdot & \cdot \\
\cdot & \cdot & \cdot \\
\cdot & \cdot & \cdot \\
x_{1 j_{m+s}}-x_{1 j_{1}} & \cdots & x_{m j_{m+s}}-x_{m j_{1}} \\
y_{1}-y_{1 j_{1}} & \cdots & y_{s}-y_{s j_{1}} \\
y_{1 j_{2}}-y_{1 j_{1}} & \cdots & y_{s j_{2}}-y_{s j_{1}} \\
\cdot & \cdot & \cdot \\
\cdot & \cdot & \cdot \\
\cdot & \cdot & \cdot \\
y_{1 j_{m+s}}-y_{1 j_{1}} & \cdots & y_{s j_{m+s}}-y_{s j_{1}}
\end{array}\right|=0
$$

where $x_{1}, \cdots, x_{m}, y_{1}, \cdots, y_{s}$ are variables, $x_{p j_{t}}(p=$ $1 \cdots, m ; t=1, \cdots, m+s)$ is the $p$ th input of the $\mathrm{DMU}_{j_{t}}$, and $y_{q j_{t}}(q=1, \cdots, s ; t=1, \cdots, m+s)$ is the $q$ th output of $\mathrm{DMU}_{j_{t}}$. Suppose that the equation 
of the afore-mentioned hyperplane is in the form of $P^{t}+\alpha=0$, where $z=\left(x_{1}, \cdots, x_{m}, y_{1}, \vdots, y_{s}\right), P$ is the gradient vector of the hyperplane, and $\alpha$ is a scalar;

Step 6. If $P$ has any component that is less than or equal to zero, proceed to Step 8; otherwise, let $w=\left(x_{1}^{w}, \cdots, x_{m}^{w}, y_{1}^{w}, \cdots, y_{s}^{w}\right)$ be defined as follows:

$$
\begin{array}{ll}
x_{i}^{w}=\max \left\{x_{i j} \mid j=1, \cdots, n\right\}, & i=1, \cdots, m, \\
y_{r}^{w}=\min \left\{y_{r j} \mid j=1, \cdots, n\right\}, & r=1, \cdots, s .
\end{array}
$$

If:

$$
\begin{aligned}
& P^{t} z_{j}+\alpha=0, \quad j \in D, \\
& P^{t} z_{j}+\alpha \leq 0, \quad j \in F-D, \\
& P^{t} w+\alpha<0,
\end{aligned}
$$

then $P^{t}+\alpha=0$ is supporting; otherwise, proceed to Step 8 .

Step 7. If at least one of $m+s$ members of $D$ is a weak efficient virtual DMU, then $P^{t}+\alpha=0$ is a weak defining hyperplane. Otherwise, it is a strong defining hyperplane;

Step 8. If another subset of $F$ with $m+s$ members can be found, go to Step 5; otherwise, stop.

\section{The proposed algorithm}

The proposed algorithm for finding the benchmarking region of efficiency derived by $\alpha$ percentage increase in the pre-efficiency value of the under-evaluated inefficient DMU with the VRS technology in the DEA is as follows:

In order to incorporate such a priori information into the process of our algorithm, we first specify $\alpha$ percentage related to the under-evaluated inefficient DMU based on value judgment.

The benchmarking region of an efficiency improvement for an inefficient DMU, $R E_{A}$, is just defined by the following equation:

$$
R E_{A}=\left\{\left(\begin{array}{c}
X_{i A}^{* T} \\
Y_{r A}^{* T}
\end{array}\right)^{T} \mid X_{i A}^{* T} \leq X_{i A}^{T}, Y_{r A}^{* T} \geq Y_{r A}^{T}\right\} .
$$

The first step of this method is to find all the defining (efficient) hyperplanes of the PPS. These hyperplanes can be found by using the algorithm proposed in $[2,3,14,18,20,22,40,41]$ (one of these papers can be applied to find the defining hyperplanes of the PPS). To check the accuracy of all these defining hyperplanes, however, we compared all of these approaches, which resulted in the same defining hyperplane (see Examples 1 and 2 in Section 4$)$. Let $P=\left\{p_{1}, \cdots, p_{2}, p_{t}\right\}$ be the set of these defining (efficient) hyperplanes. Then, consider $\mathrm{DMU}_{A}$ as an inefficient DMU under evaluation to find the new benchmarking region by increasing the efficiency (obtained from Model (2)) of $\mathrm{DMU}_{A}$ by $\alpha$ percentage. $\Omega_{1}$ is considered as a set of the extreme efficient DMUs from the PPS. Assume that $k=1$, and by setting $\overline{\mathrm{DMU}}_{A}^{(k)}$ as a projection point of $\mathrm{DMU}_{A}$ using Model (2), $p_{k}$ represents a hyperplane in which the projection point satisfied hyperplane $p_{k}$ equation.

It is, however, to be noted that if $\overline{\mathrm{DMU}}_{A}^{(k)}$ satisfies multiple hyperplanes-it is laid on the intersection of hyperplanes-we should follow the rest of the process for all of them. Following this, from $\overline{\mathrm{DMU}}_{A}^{(k)}$, we will consider $\widetilde{\mathrm{DMU}}_{A}^{(k)}$ whose efficiency is $\beta_{A}^{*}=\theta_{A}^{*}+\alpha \%$. To calculate the input/output amounts of $\widetilde{\mathrm{DMU}}_{A}(k)$, use the following formula:

$$
\begin{aligned}
\widetilde{\mathrm{DMU}}_{A}^{(k)} & =\left(\tilde{x}_{A}^{(k)}, \tilde{y}_{A}^{(k)}\right)=\left(\frac{\bar{x}_{A}^{(k)}}{\beta_{A}^{*}}, y_{A}^{(k)}\right) \\
& =\left(\frac{\theta_{A}^{*} x_{A}^{(k)}}{\beta_{A}^{*}}, y_{A}^{(k)}\right) .
\end{aligned}
$$

Suppose that $P_{k}^{\prime}$ is the hyperplane that passes through $\widehat{\mathrm{DMU}}_{A}^{(k)}$ and has the same normal vector as hyperplane $p_{k}$. By defining $\Omega_{1}$, we find the extreme efficient DMUs that lie on hyperplane $p_{k}$ so that at least one of its inputs or outputs is more than $\overline{\mathrm{DMU}}_{A}^{(k)}$ (in case of the existence of more than one DMU, follow the same process for all of them). Whether such a DMU exists or not, the following two case studies are considered:

Case 1. If such a DMU exists, consider the new DMU as $\overline{\mathrm{DMU}}_{A}^{(k+1)}$ subscript. Now, we must consider whether $\overline{\mathrm{DMU}}_{A}^{(k+1)}$ belongs to $R E_{A}$ or not:

i) If $\overline{\mathrm{DMU}}_{A}^{(k+1)}$ belongs to $R E_{A}$, suppose that hyperplane $p_{k+1}$ represents a defining efficient hyperplane in which $\overline{\mathrm{DMU}}_{A}^{(k+1)}$ satisfies hyperplane $p_{k+1}$ Equation. Consider $\widehat{\mathrm{DMU}}_{A}^{(k+1)}$, whose efficiency is $\beta_{A}^{*}=\theta_{A}^{*}+\alpha \%$. To calculate the input/output amounts of $\widetilde{\mathrm{DMU}}_{A}^{(k+1)}$, use the following formula:

$$
\begin{aligned}
\widetilde{\mathrm{DNU}}_{A}^{(k+1)} & =\left(\tilde{x}_{A}^{(k+1)}, \tilde{y}_{A}^{(k+1)}\right) \\
& =\left(\frac{x_{A}^{(k+1)}}{\beta_{A}^{*}}, y_{A}^{(k+1)}\right) .
\end{aligned}
$$

Here, we confront two cases as follows: 
a) If $\widetilde{\mathrm{DMU}}_{A}^{(k+1)}$ belongs to $R E_{A}$, suppose that hyperplane $P_{k+1}^{\prime}$ is the equation which passes through $\widetilde{\mathrm{DMU}}_{A}^{(k+1)}$ and has the same normal vector as hyperplane $p_{k+1}$; hence, start the process from the beginning;

b) If $\widetilde{\mathrm{DMU}}_{A}^{(k+1)}$ does not belong to $R E_{A}$, suppose that hyperplane $P_{k+1}^{\prime}$ is the equation which passes through $\widetilde{\mathrm{DMU}}_{A}^{(k+1)}$ and has the same normal vector as hyperplane $p_{k+1}$; then, to find a point on hyperplane $P_{k+1}^{\prime}$, use the following proposed model:

$$
\begin{array}{ll}
\max & \varphi+\varepsilon\left(1 s^{+}+1 s^{-}\right) \\
\text {s.t. } & \sum_{j=1}^{n} \lambda_{j} x_{j}=x_{A}-s^{-}, \\
& \sum_{j=1}^{n} \lambda_{j} y_{j}=\varphi y_{A}+s^{+}, \\
& \sum_{j=1}^{n} \lambda_{j}=1 \\
& \bar{U}\left(\varphi y_{A}+s^{+}\right)-\bar{V}\left(x_{A}-s^{-}\right)+\bar{u}_{o}=0 \\
\lambda_{j} \geq 0
\end{array}
$$

where $\bar{U} Y-\bar{V} X+\bar{u}_{o}=0$ is the equation of hyperplane $P_{k+1}^{\prime}$.

Theorem 1. The efficiency score of each transformed point of the extreme efficient DMUs is $\beta_{A}^{*}$.

Proof. Assume $\widetilde{\mathrm{DMU}}_{A}^{(k+1)}$ with coordinates:

$$
\left(x \widetilde{\mathrm{DMU}}_{A}^{(k+1)}, y_{\widetilde{\mathrm{DMU}}_{A}^{(k+1)}}\right)=\left(\frac{x_{A}^{(k+1)}}{\beta_{A}^{*}}, y_{A}^{(k+1)}\right),
$$

as an arbitrary transformed extreme efficient DMU that belongs to the virtual improved frontiers. Considering $\widetilde{\mathrm{DMU}}_{A}^{(k+1)}$ with the $\mathrm{BCC}$ inputoriented model, we obtain:

$$
\begin{array}{ll}
\min & \theta \widetilde{D M U}_{A}^{(K+1)}, \\
\text { s.t. } & \sum_{j=1}^{n} \lambda_{j} x_{j} \leq \theta{\widetilde{\mathrm{DMU}_{A}^{(K+1)}}}_{\widetilde{\mathrm{DMU}}_{A}^{(K+1)}} \\
& =\theta_{\widetilde{\mathrm{DMU}}_{A}^{(K+1)}}\left(\left(\frac{1}{\beta_{A}^{*}}\right) x_{A}^{(K+1)}\right),
\end{array}
$$

$$
\begin{aligned}
& \sum_{j=1}^{n} \lambda_{j} y_{j} \geq y_{\widetilde{\mathrm{DMU}}_{A}^{(K+1)}}=y_{A}^{(K+1)}, \\
& \sum_{j=1}^{n} \lambda_{j}=1, \\
& \lambda_{j} \geq 0,
\end{aligned}
$$

A feasible solution of this model can be:

$$
\begin{aligned}
& \left(\theta \widetilde{\mathrm{DMU}}_{A}^{(k+1)}=\beta_{A}^{*}, \quad \lambda_{\widetilde{\mathrm{DMU}}_{A}^{(k+1)}}=1,\right. \\
& \left.\lambda_{j}=0 \quad\left(j \neq \widetilde{\mathrm{DMU}}_{A}^{(k+1)}\right)\right) .
\end{aligned}
$$

Then, the optimal $\theta{\widetilde{\mathrm{DMU}^{(}}}_{A}^{(k+1)}$, i.e., $\theta_{\widetilde{\mathrm{DMU}}_{A}^{*}}^{(k+1)}$, is not greater than $\beta_{A}^{*} \cdot\left(\theta_{\widetilde{\mathrm{DMU}}_{A}^{*}}^{(k+1)} \leq \beta_{A}^{*}\right)$.

It will be sufficient to show that $\left(\theta_{\widetilde{\mathrm{DMU}}_{A}^{*}}^{(k+1)} \nless\right.$ $\left.\beta_{A}^{*}\right)$.

By the contradictory assumption, suppose that $\left(\theta_{\widetilde{\mathrm{DMU}}_{A}^{*}}^{(k+1)}<\beta_{A}^{*}\right)$. Hence, $\theta_{\widetilde{\mathrm{DMU}}_{A}^{*}}^{(k+1)}=\beta_{A}^{*}-\varepsilon$ for some $\varepsilon>0$. By evaluating Model (13) with this assumption, we get:

$$
\left\{\begin{aligned}
\sum_{j=1}^{n} \lambda_{j} x_{j} & \leq \theta{\widetilde{\mathrm{DMU}_{A}^{(K+1)}}}_{\widetilde{\mathrm{DMU}}_{A}^{(K+1)}} \\
& =\theta_{\widetilde{\mathrm{DMU}}_{A}^{*}}^{(K+1)}\left(\left(\frac{1}{\beta_{A}^{*}}\right) x_{A}^{(K+1)}\right) \\
& =\left(\frac{\beta_{A}^{*}-\varepsilon}{\beta_{A}^{*}}\right) x_{A}^{(K+1)} \\
& =\left(1-\frac{\varepsilon}{\beta_{A}^{*}}\right) x_{A}^{(K+1)} \\
\sum_{j=1}^{n} \lambda_{j} y_{j} & \geq y \widetilde{\mathrm{DMU}}_{A}^{(K+1)}=y_{A}^{(K+1)} \\
\sum_{j=1}^{n} \lambda_{j}=1 & \\
\lambda_{j} \geq 0 &
\end{aligned}\right.
$$

It is obvious that $\left(1-\frac{\varepsilon}{\beta_{A}^{*}}\right)<1$. In this manner, a feasible solution for $\operatorname{DMU}_{A}^{(k+1)}$ is:

$$
\begin{aligned}
& \theta_{\mathrm{DMU}_{A}^{(k+1)}}=\left(1-\frac{\varepsilon}{\beta_{A}^{*}}\right)<1, \quad \lambda_{\mathrm{DMU}_{A}^{(k+1)}}=1, \\
& \lambda_{j}=0, \quad\left(j \neq \mathrm{DMU}_{A}^{(k+1)}\right) .
\end{aligned}
$$

Consequently, $\mathrm{DMU}_{A}^{(k+1)}$ is inefficient and is in contrary to the assumption.

Therefore, we have $\theta_{\widetilde{\mathrm{DMU}}_{A}^{(k+1)}}=\beta_{A}^{*}$, thus completing the proof.

Let an optimal solution of Model (12) be $\left(\varphi^{*}, s^{-*}, s^{+*}\right)$. Based on the following theorems, this model gives us a point that firstly belongs to $R E_{A}$; secondly, this reference point of $\mathrm{DMU}_{A}$, i.e., $\left(x_{A}-s^{-*}, \varphi^{*} y_{A}+s^{+*}\right)$, dominates $\mathrm{DMU}_{A}$ and belongs to hyperplane $P_{k+1}^{\prime}$. 
Theorem 2. Given Model (12), we have:

a) $\left(x_{A}-s^{-*}, \varphi^{*} y_{A}+s^{+*}\right)$ is a dominating $\mathrm{DMU}_{A}$ and, thus, $\left(x_{A}-s^{-*}, \varphi^{*} y_{A}+s^{+*}\right)$ also belongs to $R E_{A}$;

b) $\left(x_{A}-s^{-*}, \varphi^{*} y_{A}+s^{+*}\right)$ lies on hyperplane $P_{k+1}^{\prime}$;

c) Model (12) is feasible.

\section{Proof.}

(a) Since $s^{-*} \geq 0, s^{+*} \geq 0$, and $\varphi^{*} \geq 0$, then we have $\left(x_{A}-s^{-*} \leq x_{A}\right)$ and $\left(\varphi^{*} y_{A}+s^{+*} \geq y_{A}\right)$. Considering $\mathrm{DMU}_{A}$ as an inefficient unit, the optimal solution of Model (12) is expressed as $\left(\varphi^{*}>1\right)$ or $\left[\left(s^{-*} . s^{+*}\right) \neq 0\right]$. Then, we have:

$$
\left(\begin{array}{c}
-\left(x_{A}-s^{-*}\right) \\
\varphi^{*} y_{A}+s^{+*}
\end{array}\right) \underset{\neq}{\gtrless}\left(\begin{array}{c}
-x_{A} \\
y_{A}
\end{array}\right),
$$

which means that $\left(x_{A}-s^{-*}, \varphi^{*} y_{A}+s^{+*}\right)$ is a dominating $\mathrm{DMU}_{A}$; thus, $\left(x_{A}-s^{-*}, \varphi^{*} y_{A}+\right.$ $\left.s^{+*}\right)$ also belongs to REA;

(b) Evidently, based on the fourth constraint of Model (12), the obtained reference point from Model (12), i.e., point $\left(x_{A}-s^{-*}, \varphi^{*} y_{A}+s^{+*}\right)$, lies on hyperplane $P_{k+1}^{\prime}: \bar{U} Y-\bar{V} Y+\bar{u}_{o}=0$;

(c) Considering that the extreme points used in the proposed algorithm belong to $R E_{A}$, if the extreme DMU does not belong to $R E_{A}$ in one step, the new obtained point from Model (12) will be in $R E_{A}$ in the next step of the algorithm (see Section b in Case 1). Thus, each transformed point of the extreme efficient DMUs will belong to $R E_{A}$ (if the transformed point of the extreme DMU does not belong to $R E_{A}$ in one step, then the new obtained point from Model (12) will be in $R E_{A}$ in the next step of the algorithm (see Section ii) in Case 1)).

According to the algorithm procedure, the new improved facets (the new virtual frontiers) are obtained from the convex hull of these points. Besides, PPS is convex and, thus, the convex hull of these points belongs to $R E_{A}$; consequently, the new improved facets also belong to $R E_{A}$.

This illustrates not only the fact that the fourth constraint belongs to $R E_{A}$, but also that the reference point of $\mathrm{DMU}_{A}$, i.e., $\left(x_{A}-s^{-*}, \varphi^{*} y_{A}+\right.$ $s^{+*}$ ), belongs to $R E_{A}$ (based on Part A of Theorem 2). The model is thus always feasible.

Replace the new point with $\widetilde{\mathrm{DMU}}_{A}(k+1)$ by connecting $\mathrm{DMU}_{A}$ to the new point $\widetilde{\mathrm{DMU}}_{A}^{(k+1)}$, which lies on hyperplane $P_{k+1}^{\prime}$ (see Figure 1 ); this process is finished;

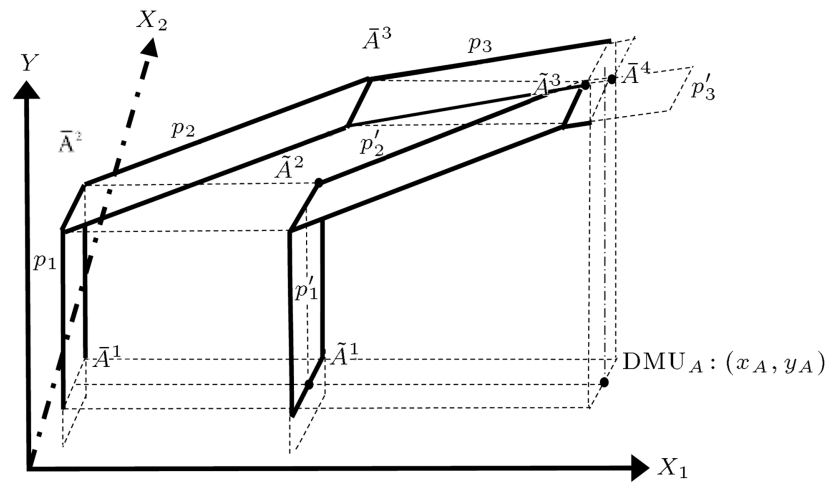

Figure 1. The benchmarking region of efficiency (Case 1).

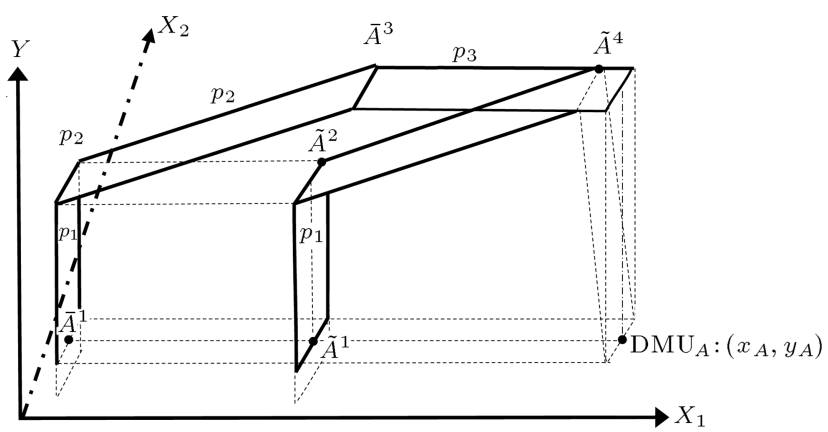

Figure 2. The benchmarking region of efficiency (Case 2 . $\widetilde{\mathrm{DMU}}_{A}^{(3)}$ belongs to $\left.R E_{A}\right)$.

ii) If $\overline{\mathrm{DMU}}_{A}^{(k+1)}$ does not belong to $R E_{A}$, then the process is the same with Part b) of Section $i$ ), except that instead of the equation of hyperplane $P_{k+1}^{\prime}$ in the fourth constraint of Model (12), the fourth constraint is replaced with hyperplane $P_{k}^{\prime}$.

Case 2. If such a unit does not exist, hyperplane $p_{k+1}$ is a weak defining hyperplane. Similarly, calculate the output/input amounts of $\widetilde{\mathrm{DMU}}_{A}^{(k+1)}$ by Eq. (11). If $\widetilde{\mathrm{DMU}}_{A}^{(k+1)}$ belongs to $R E_{A}$, then it is sufficient to connect $\mathrm{DMU}_{A}$ to $\widetilde{\mathrm{DMU}}_{A}^{(k+1)}$ (see Figure 2); otherwise, find a point from Model (12) with the equation of hyperplane $P_{k+1}^{\prime}$ in the fourth constraint.

Replace the new point with $\widetilde{\mathrm{DMU}}_{A}^{(k+1)}$ and name it $\widetilde{\mathrm{DMU}}_{A}^{(k+1)}$. It is then sufficient to connect $\mathrm{DMU}_{A}$ to $\widehat{\mathrm{DMU}}_{A}^{(k+1)}$ s (see Figure 3 ).

Evidently, $\widehat{\mathrm{DMU}}_{A}^{(k+1)}$ lies on both hyperplanes, $P_{k}^{\prime}$ and $P_{k+1}^{\prime}$. Throughout the algorithm,

$$
\widehat{\mathrm{DMU}}_{A}^{(1)} \rightarrow \widetilde{\mathrm{DMU}}_{A}^{(2)} \rightarrow \cdots \rightarrow \widehat{\mathrm{DMU}}_{A}^{(k+1)}
$$

represents the benchmarking movement in which the points $\widetilde{\mathrm{DMU}}_{A}^{(1)} \cdot \widetilde{\mathrm{DMU}}_{A}^{(2)} \cdots \cdot \widehat{\mathrm{DMU}}_{A}^{(k+1)}$ lie on hyperplanes $P_{1}^{\prime}, P_{2}^{\prime}, \cdots, P_{k+1}^{\prime}$, respectively; the set of sub- 


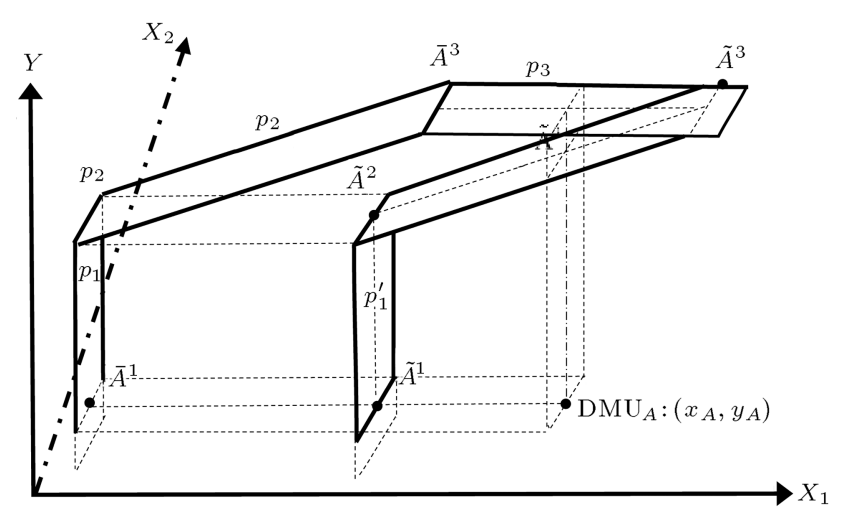

Figure 3. The benchmarking region of efficiency (Case 2. $\widetilde{\mathrm{DMU}}_{A}^{(3)}$ does not belong to $\left.R E_{A}\right)$.

scripts of the efficient improved faces of the PPS for $\mathrm{DMU}_{A}$ is considered (except the second part of Case 2 in which $\widetilde{\mathrm{DMU}}_{A}^{(k+1)}$ lies on hyperplane $P_{k}^{\prime}$ ). Note that all the new shifted units of the extreme points in the PPS have an equivalent efficiency value of $\beta_{A}^{*}=$ $\theta_{A}^{*^{(K)}}+\alpha \%$.

Hence, starting at benchmark point $\widetilde{\mathrm{DMU}}_{A}^{(1)}$, passing through all the obtained hyperplanes throughout the algorithm and, eventually, stopping at $\widetilde{\mathrm{DMU}}_{A}^{(k+1)}$, one can achieve the benchmark region of deficiency improvement.

Among the previous works, this study explores an improved benchmark and, sequentially, an improved region of efficiency for an inefficient DMU. In comparison, some papers find a benchmark turning the inefficient DMU to an efficient DMU [25]. Additionally, some other papers used the DEA sensitivity analysis just to explore a point as a virtual benchmark for an inefficient DMU [18].

Practically, an inefficient DMU sometimes cannot improve its performance to the best practice by either decreasing its inputs or increasing its outputs.

The current paper suggests an improved region that is characterized such that the efficiency value of each point in this region will be derived by the hyperplane with the same normal vector of the original PPs hyperplane. In fact, we improve all the hyperplanes that belong to $R E_{A}$. Considering that any change has implications, the process of finding a suitable benchmark is constantly faced with changes and evidently increasing costs. We, therefore, always try to remove or reduce these changes. The strength of the present approach is that, with considerably little numerical effort, one can get an insight into the improved efficiency region of a given DMU; unlike many previous works, it is based on exact results of a welldefined value judgment.

The proposed algorithm. The summary of the proposed algorithm is given below. Here, $\mathrm{DMU}_{A}$ is considered as an inefficient DMU under evaluation in order to find a new region by increasing the efficiency (obtained by Model 2) of $\mathrm{DMU}_{A}$ by $\alpha \%$.

Step 1. Find all the defining (efficient) hyperplanes of the PPS. Let $P=\left\{p_{1}, \cdots, p_{2}, p_{t}\right\}$ be the set of these defining (efficient) hyperplanes;

Step 2. Assume that $k=1$ and $\Omega_{1}=\left\{\mathrm{DMU}_{j} \mid \theta_{j}^{*}=1\right.$ and $\lambda_{j}=1$ in assessing $\mathrm{DMU}_{j}$ by Model (2) $\}$;

Step 3. Solve Model (2) and set $\overline{\mathrm{DMU}}_{A}^{(k)}$ as a projection point of $\mathrm{DMU}_{A}$;

Step 4. Look for $p_{k}$ representing a hyperplane that $\overline{\mathrm{DMU}}_{A}^{(k)}$ satisfies in hyperplane $p_{k}$ equation;

Step 5. From $\overline{\mathrm{DMU}}_{A}^{(k)}$, consider $\widetilde{\mathrm{DMU}}_{A}^{(k)}$ whose efficiency is $\beta_{A}^{*}=\theta_{A}^{*}+\alpha \%$. To calculate the input/output amounts of $\widetilde{\mathrm{DMU}}_{A}(k)$, use the following formulas:

$$
\begin{aligned}
& \text { For } \quad k=1 ; \\
& \begin{aligned}
\widetilde{\mathrm{DMU}}_{A}^{(k)} & =\left(\tilde{x}_{A}^{(k)}, \tilde{y}_{A}^{(k)}\right)=\left(\frac{\bar{x}_{A}^{(k)}}{\beta_{A}^{*}}, y_{A}^{(k)}\right) \\
& =\left(\frac{\theta_{A}^{*} x_{A}^{(k)}}{\beta_{A}^{*}}, y_{A}^{(k)}\right) .
\end{aligned}
\end{aligned}
$$

Step 6. Find the equation of hyperplane $P_{k}^{\prime}$ which passes through $\widetilde{\mathrm{DMU}}_{A}^{(k)}$ and has the same normal vector as hyperplane $p_{k}$;

Step 7. Using the definition of $\Omega_{1}$, look for extreme efficient DMUs that lie on hyperplane $p_{k}$ so that at least one of its inputs or outputs is more than $\overline{\mathrm{DMU}}_{A}^{(k)}$. (In the case of the existence of more than one DMU for all of them, follow the process from Step 8.) If such a DMU exists, go to Step 8; otherwise, go to Step 9;

Step 8. Subscript the new DMU as $\overline{\mathrm{DMU}}_{A}^{(k+1)}$. If $\overline{\mathrm{DMU}}_{A}^{(k+1)}$ belongs to $R E_{A}$, then proceed to Step 10; otherwise, proceed to Step 11;

Step 9. If such a unit does not exist, i.e., hyperplane $p_{k+1}$ is a weak defining hyperplane, calculate the output/input amounts of $\widetilde{\mathrm{DMU}}_{A}^{(k+1)}$ from Eq. (11). If $\widetilde{\mathrm{DMU}}_{A}^{(k+1)}$ belongs to $R E_{A}$, then proceed to Step 14; otherwise, proceed to Step 15;

Step 10. If $\overline{\mathrm{DMU}}_{A}^{(k+1)}$ belongs to $R E_{A}$, hyperplane $p_{k+1}$ represents a defining efficient hyperplane in which $\overline{\mathrm{DMU}}_{A}^{(k+1)}$ satisfies hyperplane $p_{k+1}$ equation. Consider $\widetilde{\mathrm{DMU}}_{A}^{(k+1)}$ whose efficiency is $\beta_{A}^{*}=\theta_{A}^{*}+\alpha \%$. 
To calculate the input/output amounts of $\widetilde{\mathrm{DMU}}_{A}^{(k+1)}$, use the following formula:

$$
\widetilde{D M U}_{A}^{(k+1)}=\left(\tilde{x}_{A}^{(k+1)}, \tilde{y}_{A}^{(k+1)}\right)=\left(\frac{x_{A}^{(k+1)}}{\beta_{A}^{*}}, y_{A}^{(k+1)}\right)_{(14)}
$$

Here, we confront two cases: either $\widetilde{\mathrm{DMU}}_{A}^{(k+1)}$ belongs to $R E_{A}$, then proceed to Step 12; or it does not belong to $R E_{A}$, then proceed to Step 13;

Step 11. If $\overline{\mathrm{DMU}}_{A}^{(k+1)}$ does not belong to $R E_{A}$, then, by using Model (12), except that instead of the equation of hyperplane $P_{k+1}^{\prime}$ in the fourth constraint of Model (12), the fourth constraint is replaced with the equation of hyperplane $P_{k}^{\prime}$.

Replace the new point with $\widetilde{\mathrm{DMU}}_{A}^{(k+1)}$ and name it $\widetilde{\mathrm{DMU}}_{A}^{(k+1)}$. By connecting $\mathrm{DMU}_{A}$ to the new point, $\widehat{\mathrm{DMU}}_{A}^{(k+1)}$, which lies on hyperplane $P_{k}^{\prime}$ and has at least one input/output value of $\mathrm{DMU}_{A}$, this process is finished. Proceed to Step 16;

Step 12. If $\widetilde{\mathrm{DMU}}_{A}^{(k+1)}$ belongs to $R E_{A}$, suppose that hyperplane $P_{k+1}^{\prime}$ is the equation that passes through $\widetilde{\mathrm{DMU}}_{A}^{(k+1)}$ and has the same normal vector as hyperplane $p_{k+1}$; hence, proceed to Step 7;

Step 13. If $\widetilde{\mathrm{DMU}}_{A}^{(k+1)}$ does not belong to $R E_{A}$, suppose that hyperplane $P_{k+1}^{\prime}$ is the equation which passes through $\widetilde{\mathrm{DMU}}_{A}(k+1)$ and has the same normal vector as hyperplane $p_{k+1}$; then, find a point from Model (12) in which the fourth constraint is the equation of hyperplane $P_{k+1}^{\prime}$. Replace the new point with $\widetilde{\mathrm{DMU}}_{A}^{(k+1)}$. By connecting $\mathrm{DMU}_{A}$ to the new point, $\widetilde{\mathrm{DMU}}_{A}(k+1)$, which lies on hyperplane $P_{k+1}^{\prime}$, this process is finished;

Step 14. If $\widetilde{\mathrm{DMU}}_{A}^{(k+1)}$ belongs to $R E_{A}$, then it is sufficient to connect $\mathrm{DMU}_{A}$ to $\widetilde{\mathrm{DMU}}_{A}^{(k+1)}$. Proceed to Step 16;

Step 15. If $\widetilde{\mathrm{DMU}}_{A}^{(k+1)}$ does not belong to $R E_{A}$, find a point from Model (12) with the equation of hyperplane $P_{k+1}^{\prime}$ in the fourth constraint. Replace the new point with $\widetilde{\mathrm{DMU}}_{A}^{(k+1)}$ and name it $\widetilde{\mathrm{DMU}}_{A}^{(k+1)}$. Then, it is sufficient to connect $\mathrm{DMU}_{A}$ to $\widetilde{\mathrm{DMU}}_{A}^{(k+1)}$;

Step 16. Stop.

The PPS is a polyhedral convex set whose vertices correspond to the efficient DMUs. Besides, a polyhedron is defined as the intersection of a finite collection of generalized halfspaces. More generally, a half space is either of the two parts into which a hyperplane divides an affine space. Since the number of the defining hyperplane of a PPS is finite, the number of the new improved hyperplanes is less than that of the defining hyperplanes. The validity of the algorithm is, therefore, obvious owing to the finite number of defining hyperplanes.

\section{Numerical example}

This section provides a numerical example that helps clarify the concept of the improved region of the DMU's efficiency and its comparative advantage over conventional DEA models.

In light of the growing strategic importance of the function of managing a company's most important asset-its reputation-there is a need of fundamentally evaluating the improved region of the DMU's efficiency to obtain a guideline for future planning.

Example 1: Let us assume that we have eight sample DMUs, each using one input to produce one output with the data given in Table 1 . All the virtual and real DMUs are depicted in Figure 4. By the foregone algorithm.

For finding the improved region by increasing the efficiency (obtained from Model (2)) of $\mathrm{DMU}_{G}$ by $\alpha \%=13.75 \%$, we first find all the maker hyperplanes of PPS. These hyperplanes' set is as follows:

$$
\{-x+2=0, y-4 x+6=0,2 y-x-9=0, y-8=0\} .
$$

Table 1. Sample DMUs.

\begin{tabular}{lcccccccc}
\hline DMU & $\boldsymbol{A}$ & $\boldsymbol{B}$ & $\boldsymbol{C}$ & $\boldsymbol{D}$ & $\boldsymbol{E}$ & $\boldsymbol{F}$ & $\boldsymbol{G}$ & $\boldsymbol{H}$ \\
\hline Input & 2 & 3 & 7 & 4.4 & 3.3 & 6 & 4 & 8 \\
Output & 2 & 6 & 8 & 6 & 4.5 & 5 & 3 & 4 \\
\hline
\end{tabular}

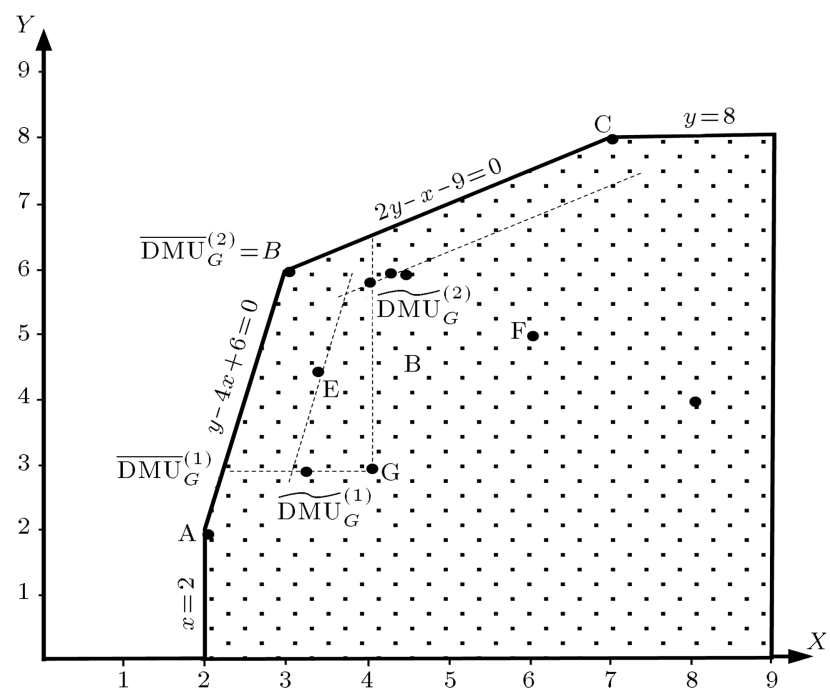

Figure 4. Efficient frontiers of PPS. 
$\Omega_{1}=\left\{\mathrm{DMU}_{A}, \mathrm{DMU}_{B}, \mathrm{DMU}_{C}\right\}$ is the set of the extreme efficient DMUs of the data of Table 1.

By solving Model (2) and setting $\overline{\mathrm{DMU}}_{G}^{(1)}$ as the projection point of $\mathrm{DMU}_{G}$, we have:

$$
\overline{\mathrm{DMU}}_{G}^{(1)}=(\bar{x}, \bar{y})=(2.25,3) .
$$

From $\overline{\mathrm{DMU}}_{G}^{(1)}$, which lies on $p_{1}: y-4 x+6=0$, we are considering $\widehat{\mathrm{DMU}}_{G}^{(1)}$, whose efficiency is:

$$
\theta_{G}^{*}=0.5685 \stackrel{\alpha \%=13.75 \%}{\stackrel{2}{\longrightarrow}} \beta_{G}^{*}=\theta_{G}^{*}+\alpha \%=0.7 .
$$

Using Eq. (10), we calculate the input/output amounts of $\widehat{\mathrm{DMU}}_{G}^{(1)}$ :

$$
\widetilde{\mathrm{DMU}}_{G}^{(1)}=\left(\tilde{x}_{G}^{(1)}, \tilde{y}_{G}^{(1)}\right)=(3.2143,3) .
$$

Now, we are looking for hyperplane $p_{1}^{\prime}$, which passes through $\widetilde{\mathrm{DMU}}_{G}^{(1)}$ and has the same normal vector as hyperplane $p_{1}$; this is shown as follows:

$$
y-4 x+12.857=0 .
$$

$\mathrm{DMU}_{B}$ is an extreme efficient DMU that lies on hyperplane $p_{1}$; its inputs and outputs are more than $\overline{\mathrm{DMU}}_{G}^{(1)}$. $\overline{\mathrm{DMU}}_{G}^{2}$.

Since $\mathrm{DMU}_{B}$ belongs to $R E_{G}$, we subscript it as

A defining efficient hyperplane in which $\overline{\mathrm{DMU}}_{A}^{2}$ satisfies hyperplane $p_{2}$ equation, is represented by $p_{2}=$ $2 y-x-9=0$. By Eq. (11), we calculate $\widehat{\mathrm{DMU}}_{G}^{2}$, whose efficiency is $\beta=0.7$, as follows:

$$
\widetilde{\mathrm{DMU}}_{G}^{2}=\left(\tilde{x}_{G}^{2}, \tilde{y}_{G}^{2}\right)=(4.2857,6) \text {. }
$$

The equation of the hyperplane which passes through $\widetilde{\mathrm{DMU}}_{G}^{(2)}$ and has the same normal vector as hyperplane $p_{2}$ is as follows:

$$
P_{2}^{\prime}=2 y-x-7.7143=0,
$$

since $\widetilde{\mathrm{DMU}}_{G}^{2}$ does not belong to $R E_{G}$, based on Step 13 of the algorithm, we replace $\widetilde{\mathrm{DMU}}_{G}^{2}$ with the new point (4,5/85715) obtained from Model (12), which lies on hyperplane $P_{2}^{\prime}$ and has at least one input/output value of $\mathrm{DMU}_{G}$ (see Figure 4).

Since there is no other DMU in $R E_{G}$, there are also no other improved hyperplanes.
Example 2: The data of 9 DMUs from Hosseinzadeh Lotfi's paper (2011) [19] are used as a numerical example and consist of two inputs and one output, as shown in Table 2.

We intend to find the improved region of the DMU's efficiency using the data in Table 2. We, therefore, use the proposed algorithm to obtain the improved efficiency region of $\mathrm{DMU}_{2}$ by increasing its current efficiency value $\left(\theta_{2}^{*}=0.6885246\right.$ to $\alpha \%=$ $11 / 1475 \%$ ).

After finding all the defining hyperplanes as shown in Figure 5, the set of extreme efficient points of PPS is as follows:

$$
\Omega_{1}=\left\{\mathrm{DMU}_{3}, \mathrm{DMU}_{4}, \mathrm{DMU}_{5}, \mathrm{DMU}_{7}\right\}
$$

By determining the radial projection reference point of $\mathrm{DMU}_{2}$ by Model (2) $\overline{\mathrm{DMU}}_{2}^{(1)}$, we look for hyperplane $p_{1}$ in which $\overline{\mathrm{DMU}}_{2}^{(1)}$ satisfies hyperplane $p_{1}$ equation:

$$
\begin{aligned}
& \begin{aligned}
& \mathrm{DMU}_{2}=\left(x_{1}, x_{2}, y\right)=(7,3,4) \rightarrow \overline{\mathrm{DMU}}_{2}^{(1)} \\
&=(0.6885246(7), 0.6885246(3), 4), \rightarrow \overline{\mathrm{DMU}}_{2}^{(1)} \\
&=(4.8196722,2.0655738,4), \\
& p_{1}: 0.352941 y-0.411765 x_{1}-0.235294 x_{2}+1.06=0 .
\end{aligned}
\end{aligned}
$$

Now, we intend to find $\widetilde{\mathrm{DMU}}_{2}^{(1)}$, whose efficiency is $\theta_{2}^{*}+\alpha \%=0.8$. The input/output amounts of $\widetilde{\mathrm{DMU}}_{2}^{(1)}$ are calculated as follows:

$$
\begin{aligned}
\widetilde{\mathrm{DMU}}_{2}^{(1)} & =\left(\tilde{x}_{12}^{(1)}, \tilde{x}_{22}^{(1)}, \tilde{y}_{2}^{(1)}\right) \\
& =\left(\frac{0.6885246(7)}{0.8}, \frac{0.6885246(3)}{0.8}, 4\right) \\
& =(6.02459025,2.58196725,4) .
\end{aligned}
$$

In Step 6, we find the equation of hyperplane $P_{1}^{\prime}$ which passes through $\widetilde{\mathrm{DMU}}_{2}^{(1)}$ and has the same normal vector as hyperplane $p_{1}$ which is as follows:

$$
p_{1}=(\alpha, \beta, \gamma)=(0.352941,-0.411765,-0.235294)
$$

Table 2. The data of the eight DMUs.

\begin{tabular}{cccccccccc}
\hline & $\mathbf{D M U}_{\mathbf{1}}$ & $\mathbf{D M U}_{\mathbf{2}}$ & $\mathbf{D M U}_{\mathbf{3}}$ & $\mathbf{D M U}_{\mathbf{4}}$ & $\mathbf{D M U}_{\mathbf{5}}$ & $\mathbf{D M U}_{\mathbf{6}}$ & $\mathbf{D M U}_{\mathbf{7}}$ & $\mathbf{D M U}_{\mathbf{8}}$ & $\mathbf{D M U}_{\mathbf{9}}$ \\
\hline Input 1 & 4 & 7 & 8 & 4 & 2 & 10 & 12 & 10 & 13 \\
Input 2 & 3 & 3 & 1 & 2 & 4 & 1 & 1 & 1.5 & 2 \\
Output & 2 & 4 & 7 & 3 & 2 & 5 & 8 & 7 & 7.5 \\
\hline
\end{tabular}




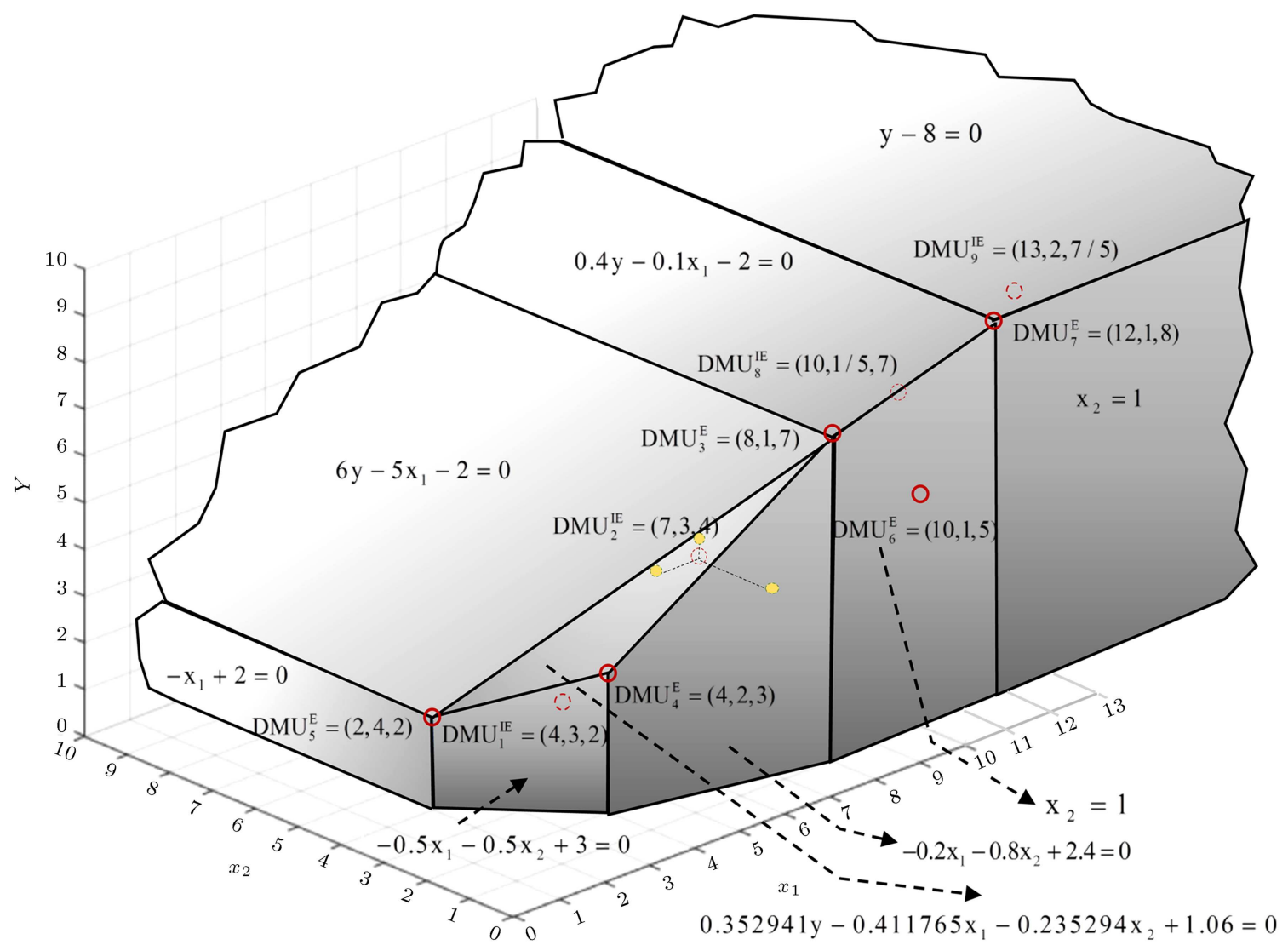

Figure 5. The visual description scheme.

$$
\begin{aligned}
\alpha\left(y-y_{A}\right)+\beta\left(x_{1}-x_{1 A}\right)+\gamma\left(x_{2}-x_{2 A}\right)=0 \rightarrow \\
0.352941(y-4)-0.411765(y-4) \\
-0.411765\left(x_{1}-6.02459025\right) \\
-0.235294\left(x_{2}-2.58196725\right)=0, \\
P_{1}^{\prime}: 0.352941 y-0.411765 x_{1}-0.235294 x_{2} \\
+1.676472806=0 .
\end{aligned}
$$

$\mathrm{DMU}_{3}$ and $\mathrm{DMU}_{5}$ are the extreme efficient DMUs lying on hyperplane $p_{1}$ where at least one of their inputs or outputs is more than $\overline{\mathrm{DMU}}_{2}^{(1)}$. Based on Step 11 of the algorithm, since $\overline{\mathrm{DMU}}_{2}^{(2)}$ does not belong to $R E_{2}$, we find point $\widetilde{\mathrm{DMU}}_{A}^{(2)}=(7,2.4687495,5.062504)$ by Model (12) in which the fourth constraint is the equation of hyperplane $P_{1}^{\prime}$.

At this point, to clarify the strategy of the proposed algorithm, we follow the algorithm for $\mathrm{DMU}_{9}=$ $(13,2,7.5)$ as follows.

We solve Model (2) and find that $\overline{\mathrm{DMU}}_{9}^{(1)}$ is a projection point of $\mathrm{DMU}_{9} ; \overline{\mathrm{DMU}}_{9}^{(1)}=(10,1.5,7.5)$.

The equation of a hyperplane is $p_{1}: 0.4 y-0.1 x 1-$ $2=0$, such that $\overline{\mathrm{DMU}}_{9}^{(1)}$ satisfies its equation.
Now, we consider $\widetilde{\mathrm{DMU}}_{9}^{(1)}$ whose efficiency is $\beta_{9}^{*}=$ $\theta_{9}^{*}+\alpha \%=0.769230769+0.130769231=0.9(\alpha \%=$ $13.0769231 \%$ ).

By Eq. (10), the following unit is calculated:

$$
\widetilde{\mathrm{DMU}}_{9}^{(1)}=(11 . \overline{1}, 1.709401709,7.5)
$$

The equation of hyperplane $P_{1}^{\prime}$ is $0.4 y-0.1 x_{1}-1 . \overline{8}=0$

The extreme efficient DMU that lies on hyperplane $p_{1}$ is $\mathrm{DMU}_{7}=\overline{\mathrm{DMU}}_{9}^{(2)}$ and its first input is greater than $\overline{\mathrm{DMU}}_{9}^{(1)}$.

As $\overline{\mathrm{DMU}}_{9}^{(2)}$ belongs to $R E_{9}$, based on Step 10 of the proposed algorithm, we find two defining efficient hyperplanes, $p_{2}$ and $p_{3}$, in which $\overline{\mathrm{DMU}}_{9}^{(2)}$ satisfies their equations as follows:

$$
\left\{\begin{array}{l}
p_{2}: y-8=0 \\
p_{3}: x_{2}-1=0
\end{array}\right.
$$

Now, by using Eq. (11), $\widetilde{\mathrm{DMU}}_{9}^{(2)}$ is considered, whose efficiency is $\beta_{9}^{*}=0.9: \widehat{\mathrm{DMU}}_{9}^{(2)}=(13 . \overline{3}, 1 . \overline{1}, 8)$.

As $\widetilde{\mathrm{DMU}}_{9}^{(2)}$ does not belong to $R E_{9}$, suppose that $P_{2}^{\prime}: y-8=0, P_{3}^{\prime}: x_{2}-1 . \overline{1}=0$ are the equations of the hyperplanes that pass through $\widetilde{\mathrm{DMU}}_{9}^{(2)}$ and have 
the same normal vector as hyperplanes $p_{2}$ and $p_{3}$, respectively.

In addition, there is no other extreme efficient unit that passes through equations of hyperplanes $p_{2}$ and $p_{3}$. Based on Step 13, to understand the improved region better, Model (12) is employed. In the equation of $y-8=0$, the obtained point is $(9.7 \overline{3}, 1.2 \overline{6}, 8.0000325)$; in the equation of $x_{2}-1 . \overline{1}=0$, the obtained point is $(13,1.2,8.6750025)$. We replace the new points with $\widehat{\mathrm{DMU}}_{9}^{(2)}$ and $\widetilde{\mathrm{DMU}}_{9}^{(3)}$. By connecting $\mathrm{DMU}_{9}$ to the new points, $\widetilde{\mathrm{DMU}}_{9}^{(2)}$ and $\widetilde{\mathrm{DMU}}_{9}^{(3)}$, which lie on hyperplanes $P_{2}^{\prime}$ and $P_{3}^{\prime}$, the process is finished. The part of improved obtained hyperplanes $P_{1}^{\prime}, P_{2}^{\prime}$, and $P_{3}^{\prime}$, included in the points $\widetilde{\mathrm{DMU}}_{9}^{(1)}, \widetilde{\mathrm{DMU}}_{9}$, and $\widetilde{\mathrm{DMU}}_{9}^{(3)}$, is the improvement benchmarking region.

Note that, in Figure 5, notations $\mathrm{DMU}_{j}^{E}$ and $\mathrm{DMU}_{j}^{I E}, j=1, \cdots, n$, respectively, represent the efficient and inefficient units.

\section{Conclusions}

As it is an issue of considerable importance, this study sought to make the standards more flexible for choosing the benchmarks. This standard can, however, differ from that of decision-makers. If a company has a specific corporate strategy or future vision, the standards for choosing benchmarks might be more complex. There is a central question in the evaluation of an inefficient DMU: instead of having a benchmark with an efficiency value of one, how can an inefficient DMU achieve a more realistic benchmark in which the efficiency is less than one? This means that a wide range of knowledge on this study becomes available. A framework for defining a benchmark region in evaluating an inefficient DMU was derived from the value judgment by increasing the current efficiency. This paper developed a procedure for shifting the efficient frontier toward the inefficient DMU. The procedure yielded an exact improved region that is derived by increasing the efficiency score of a specific inefficient DMU by $\alpha \%$, and the efficiency score of each point belonging to the new improved frontiers is different. It is indeed a new virtual frontier that is recognized as a realistic benchmarking region for the under-evaluated inefficient DMU. One of the advantages of the current analysis is the incorporation of value judgment into the current situation of hyperplanes through specific efficiency improvement for the under-evaluated inefficient DMU. The value judgment here can, therefore, be represented by the improved region concept. In addition, we used the sensitivity analysis of efficiency to find the new region. Further research will concentrate on the region of the MPSS (Most Productive Scale Size) by using hyperplanes to evaluate DMUs.

\section{References}

1. Charnes, A., Cooper, W.W., and Rhodes, E. "Measuring the efficiency of decision making units", European Journal of Operational Research, 2, pp. 429-444 (1978).

2. Tavana, M. and Khalili-Damghani, K. "A new twostage Stackelberg fuzzy data envelopment analysis model", Measurement, 53, pp. 277-296 (2014).

3. Banihashemi, S., Tohidi, G., and Sanei, M. "Sensitivity analysis of efficient and inefficient units in integer-valued data envelopment analysis", International Journal of Mathematical Modelling \& Computations, 4, pp. 45-53 (2014).

4. Banker, R.D., Charnes, A., and Cooper, W.W. "Some models for estimating technical and scale inefficiencies in data envelopment analysis", Management Science, 30, pp. 1078-1092 (1984).

5. Bogetoft, P. and Hougaard, J.L. "Efficiency evaluations based on potential (non-proportional) improvements", Journal of Productivity Analysis, 12, pp. 233247 (1999).

6. Diabat, A., Shetty, U., and Pakkala, T. "Improved efficiency measures through directional distance formulation of data envelopment analysis", Annals of Operations Research, 229, pp. 325-346 (2015).

7. Kao, C. "Efficiency improvement in data envelopment analysis", European Journal of Operational Research, 73, pp. 487-494 (1994).

8. Chen, T.-H., Bao, C.-P., and Chang, S.-Y. "Another view of efficiency improvement in data envelopment analysis", Journal of the Chinese Institute of Industrial Engineers, 26, pp. 109-114 (2009).

9. Khoshfetrat, S. and Daneshvar, S. "Improving weak efficiency frontiers in the fuzzy data envelopment analysis models", Applied Mathematical Modelling, 35, pp. 339-345 (2011).

10. Akbarian, D. "On characterizing weak defining hyperplanes (weak Facets) in DEA with constant returns to scale technology", arXiv preprint arXiv:1409.7546, (2014).

11. Hadi-Vencheh, A., Jablonsky, J., and Esmaeilzadeh, A. "The slack-based measure model based on supporting hyperplanes of production possibility set", Expert Systems with Applications, 42, pp. 6522-6529 (2015).

12. Jahanshahloo, G.R., Lotfi, F.H., and Zohrehbandian, M. "Finding the piecewise linear frontier production function in data envelopment analysis", Applied Mathematics and Computation, 163, pp. 483-488 (2005).

13. Korhonen, P. "Searching the efficient frontier in data envelopment analysis", In Aiding Decisions with Multiple Criteria, Springer, pp. 543-558 (2002).

14. Jahanshahloo, G.R., Hosseinzadeh, F., Rezai, H.Z., and Balf, F.R. "Finding strong defining hyperplanes of production possibility set", European Journal of Operational Research, 177, pp. 42-54 (2007). 
15. Yu, P. and Zeleny, M. "The set of all nondominated solutions in linear cases and a multicriteria simplex method", Journal of Mathematical Analysis and Applications, 49, pp. 430-468 (1975).

16. Hosseinzadeh, F., Jahanshahloo, G.R., Mozaffari, M., and Gerami, J. "Finding DEA-efficient hyperplanes using MOLP efficient faces", Journal of Computational and Applied Mathematics, 235, pp. 1227-1231 (2011).

17. Charnes, A., Cooper, W.W., Lewin, A.Y., Morey, R.C., and Rousseau, J. "Sensitivity and stability analysis in DEA", Annals of Operations Research, 2, pp. 139-156 (1984).

18. Forghani, A. and Najafi, E. "Sensitivity analysis in two-stage DEA", Iranian Journal of Optimization, 07, pp. 872-886 (2015).

19. Khalili-Damghani, K. and Taghavifard, B. "Sensitivity and stability analysis in two-stage DEA models with fuzzy data", International Journal of Operational Research, 17, pp. 1-37 (2013).

20. Huang, Z., Li, S., and Rousseau, J. "Determining rates of change in data envelopment analysis", Journal of the Operational Research Society, 48, pp. 591-593 (1997).

21. Jahanshahloo, G.R., Hosseinzadeh, F., Shoja, N., Sanei, M., and Tohidi, G. "Sensitivity and stability analysis in DEA", Applied Mathematics and Computation, 169, pp. 897-904 (2005).

22. Ebrahimkhani, N., Hosseinzadeh, F., RostamyMalkhalifeh, M., Jahanshahloo, G., and Namin, M.A. "An improved approach on sensitivity and stability analysis in DEA", International Journal of Applied Mathematics and Statistics ${ }^{T M}, \mathbf{5 6}$, pp. 75-84 (2017).

23. Kordrostami, S., Pourjafar, S., and Pourjafar, H. "A geometric method in data envelopment analysis to obtain the region of efficiency", Indian Journal of Science and Technology, 6, pp. 4116-4122 (2013).

24. Zamani, P. and Borzouei, M. "Finding stability regions for preserving efficiency classification of variable returns to scale technology in data envelopment analysis", Journal of Industrial Engineering International, 12, pp. 499-507 (2016).

25. Farrell, M.J. "The measurement of productive efficiency", Journal of the Royal Statistical Society. Series A (General), 120, pp. 253-290 (1957).

26. Kerstens, K. and Vandenâ Eeckaut, P., Technical Efficiency Measures on DEA and FDH: A Reconsideration of the Axiomatic Literature, In Université Catholique de Louvain, Center for Operations Research and Econometrics (CORE) (1995).

27. Colwell, R.J. and Davis, E.P. "Output and productivity in banking", The Scandinavian Journal of Economics, S111-S129 (1992).
28. Lovell, C.K. and Schmidt, P. "A comparison of alternative approaches to the measurement of productive efficiency", In Applications of Modern Production Theory: Efficiency and Productivity, Springer, pp. 3-32 (1988).

29. Pahlavan, R., Omid, M., and Akram, A. "Application of data envelopment analysis for performance assessment and energy efficiency improvement opportunities in greenhouses cucumber production", Journal of Agricultural Science and Technology, 14, pp. 1465-1475 (2012).

30. Coelli, T. "A multi-stage methodology for the solution of orientated DE models", Operations Research Letters, 23, pp. 143-149 (1998).

31. Portela, M.C.A.S., Borges, P.C., and Thanassoulis, E. "Finding closest targets in non-oriented DEA models: the case of convex and non-convex technologies", Journal of Productivity Analysis, 19, pp. 251-269 (2003).

32. Post, T. and Spronk, J. "Performance benchmarking using interactive data envelopment analysis", European Journal of Operational Research, 115, pp. 472487 (1999).

33. Russell, R.R. "Measures of technical efficiency", Journal of Economic Theory, 35, pp. 109-126 (1985).

34. Frei, F.X. and Harker, P.T. "Projections onto efficient frontiers: theoretical and computational extensions to DEA", Journal of Productivity Analysis, 11, pp. 275300 (1999).

35. Golany, B., Phillips, F., and Rousseau, J. "Models for improved effectiveness based on DEA efficiency results", IIE Transactions, 25, pp. 2-10 (1993).

36. Baek, C. and Lee, J.-D. "The relevance of DEA benchmarking information and the least-distance measure", Mathematical and Computer Modelling, 49, pp. 265275 (2009).

37. Sayin, S. "An algorithm based on facial decomposition for finding the efficient set in multiple objective linear programming", Operations Research Letters, 19, pp. 87-94 (1996).

38. Jahanshahloo, G., Lotfi, F.H., and Akbarian, D. "Finding weak defining hyperplanes of PPS of the BCC model", Applied Mathematical Modelling, 34, pp. 3321-3332 (2010).

39. Seiford, L.M. and Zhu, J. "Context-dependent data envelopment analysis-measuring attractiveness and progress", Omega, 31, pp. 397-408 (2003).

40. Sh. Banihashemi, M.S., Abri, A.GH., and Sourani, F. "Finding 'Improvement region' for the inefficient units in data envelopment analysis", Applied Mathematics \& Information Sciences, Appl. Math. Inf. Sci., 10, pp. 1169-1176 (2016).

41. Cherchye, L. and Van Puyenbroeck, T. "A comment on multi-stage DEA methodology", Operations Research Letters, 28, pp. 93-98 (2001). 


\section{Biographies}

Nasrin Ebrahimkhani Ghazi is a PhD Student at the Mathematics Department of the Islamic Azad University, Sciences and Research Branch, Tehran, Iran. Her research interests include data envelopment analysis and operation research.

Farhad Hosseinzadeh Lotfi is a Professor at the Department of Mathematics, Science and Research Branch, Islamic Azad University, Tehran, Iran. His major research interests are operations research and data envelopment analysis. His publications have appeared in several journals, including European Journal of Operational Research, Computers Industrial Engineering, Journal of the Operational Research Society, Applied Mathematics and Computation, Applied Mathematical Modelling, Mathematical and Computer Modelling, and Journal of the Operational Research Society of Japan, among others.

Mohsen Rostamy-Malkhalifeh is an Associate Professor of the Department of Mathematics, Science and Research Branch, Islamic Azad University, Tehran,
Iran. His major research interests are operations research and data envelopment analysis.

Gholamreza Jahanshahloo is a Professor at the Department of Mathematics, Teacher Training University, Tehran, Iran. He also published more than 100 papers in national and international journals. Approximately $60 \mathrm{PhD}$ students have graduated under his supervision. His fields of research interest are operations research and data envelopment analysis. His publications have appeared in several journals, including European Journal of Operational Research, Computers Industrial Engineering, Journal of the Operational Research Society, Applied Mathematics and Computation, Applied Mathematical Modelling, Mathematical and Computer Modelling, and Journal of the Operational Research Society of Japan, among others.

Mahnaz Ahadzadeh Namin is an Assistant Professor at the Department of Mathematics, Shahr-e -Qods Branch, Islamic Azad University, Tehran, Iran. Her research interests include operations research and data envelopment analysis. 